\title{
Labyrinthe
}

32 | 2009 (1)

Le petit théâtre intellectuel

\section{Sabina Guzzanti, ou La Scandaleuse opiniâtre}

\section{Renaud Pasquier}

\section{(2) OpenEdition}

\section{Journals}

Édition électronique

URL : http://journals.openedition.org/labyrinthe/3998

DOI : $10.4000 /$ labyrinthe.3998

ISSN : 1950-6031

Éditeur

Hermann

Édition imprimée

Date de publication : 19 juin 2009

Pagination : 89-92

ISBN : 978-2-7056-6885-3

Référence électronique

Renaud Pasquier, « Sabina Guzzanti, ou La Scandaleuse opiniâtre », Labyrinthe [En ligne], 32 | 2009 (1),

mis en ligne le 01 février 2011, consulté le 01 mai 2019. URL : http://journals.openedition.org/

labyrinthe/3998; DOI : 10.4000/labyrinthe.3998

Propriété intellectuelle 


\section{BEPPE GRILLO, OU L'IMPRÉCATEUR CONNECTÉ}

Il y a vingt ans j'ai tourné une publicité. Mais ensuite j'ai compris certaines choses sur la publicité. Il y a quinze ans je m'en prenais aux politiciens. Mais ensuite j'ai compris certaines choses sur l'économie. Il y a dix ans je finissais mes spectacles en bousillant un ordinateur à coups de masse. Mais ensuite j'ai compris certaines choses sur les ordinateurs et sur Internet. Aujourd'hui la publicité me paraît l'un de nos maux principaux, l'économie, la vraie patronne de la politique, et Internet l'un des rares espoirs qui demeurent pour se défendre et redonner à la politique la place que l'économie lui a volée. Le 26 janvier j'ai ouvert un blog sans bien savoir ce que c'était. Je commence à comprendre maintenant ${ }^{1} \ldots$

Récit d'une révélation, et plus que cela: Beppe Grillo est, avant tout, un Converti. Porte-parole vociférant des consommateurs spoliés et des citoyens aliénés, il s'acharnait, devant des salles combles, dans ses longs monologues des années quatre-vingt-dix, sur les grandes entreprises (Telecom Italia fut sa cible privilégiée) avides de contrôle et destructrices de l'environnement, sur leurs valets politiciens et journalistes, et plus largement sur notre désolante société de consommation - l'informatique en était le symbole et la pointe. Il n'a certes perdu ni verve ni colère, mais il a vu la Lumière, qui est le Réseau. Le voilà, hirsute Janus moderne, doté de deux visages de Pythies: Cassandre, il déplore le désastre environnant, et ne voit que cendres à l'horizon («Les jeunes me demandent: "quel futur avons-nous?" Les enfants, ne vous tracassez par pour le futur: vous n'en avez pas. Un souci en moins $\left.{ }^{2} »\right)$; Benoîte, il chante la

\footnotetext{
1. http://www.beppegrillo.it/2008/08/stiamo_vincendo_e_loro_lo_sanno/index. html Beppe Grillo, « Ho visto il futuro » [« J'ai vu le futur »], Internazionale, mai 2005 (ma traduction, de même pour toutes les citations suivantes).

2. http://www.beppegrillo.it/2008/12/discorso_di_capodanno/index. html (consulté le 02/02/09, de même pour les citations qui suivent).
} 
démocratie directe à venir, transparente et radieuse (« le futur est beauté, et eux n'en font pas partie $\left.{ }^{1} »\right)$.

Pour beaucoup, chez l'actuel Blogueur imprécateur, il n'est plus rien du Comique «national-populaire » des années quatre-vingt, vedette du petit et du grand écran, nimbé d'une aura d'impertinence pour avoir subi les foudres du Pouvoir (par la main de Bettino Craxi, alors omnipotent Président du Conseil, bien avant la condamnation et l'exil tunisien jusqu'à la mort). Concédons qu'il apparaît plus télévangéliste qu'amuseur public, lorsque bras en croix, la voix altérée, les yeux dans le vague, il égrène les métaphores :

C'est VOUS qui devez reprendre en main votre vie. Recommencez à faire de la politique, chaque jour. Au supermarché, à l'école, au travail, au feu rouge, dans la nature, dans votre immeuble. Il n'y a personne de l'autre côté du mur. Frappez, la porte restera fermée. Ne croyez plus aux journaux et aux télévisions. Ils mentent, elles mentent. Banques, médias, politique, grandes entreprises, c'est la même chose. Les mêmes personnes. Un monstre qui dévore le Pays, qui vous fait croire ce qu'il veut ${ }^{2}$.

Le prédicateur aurait tué l'amuseur? Parions plutôt qu'il l'augmente et le complète, mais ne l'efface pas. C'est toujours le Comique, son indéniable talent, qui attire les foules, et prête sa puissance au Blogueur, qui lui offre en échange un auditoire sans égal. Car le blog de Grillo n'est pas une simple vitrine égotiste, mais l'efficace support d'un véritable mouvement de masse. Qu'il devînt très vite le plus visité d'Italie, et mondialement connu, la chose était notable, curieuse. Mais le Réseau de Grillo n'est pas seulement virtuel, beaucoup le découvrirent avec effarement le 8 septembre 2007, à Bologne lors du premier et impressionnant (60000 personnes) rassemblement de ceux qu'on appellera désormais les grillini (ou grillisti, c'est selon), le premier $V$-Day: V pour Victoire, pour Vendetta ${ }^{3}$, et surtout pour Vaffanculo! («Va te faire foutre!»). Or il ne s'agissait pas d'applaudir un spectacle de Grillo, mais de défendre la loi d'initiative populaire baptisée " Parlement propre ». Le blog ne charriait

\footnotetext{
1. http://www.beppegrillo.it/2008/08/stiamo_vincendo_e_loro_lo_sanno/index. html.

2. http://www.beppegrillo.it/2007/09/piazza_maggiore/index. html.

3. Le sigle du V-Day est emprunté à la bande dessinée d'Alan Moore (ou à son adaptation cinématographique) et à « $V$ », son héros terroriste anarchiste.
} 
donc pas seulement visiteurs, clics ou commentaires: il tissait les liens d'une communauté, qui ne se contente pas de grandes manifestations, mais s'organise autour de rencontres périodiques, les meet-up (le nom du réseau social en ligne qui permet l'organisation de ces réunions), et d'actions citoyennes diverses dont le blog se fait l'écho et le promoteur.

Le mot de Gourou vint alors sur des lèvres ricaneuses - qu'atteste aussi la tignasse omniprésente de Grillo, devenu emblème et logo. D'autres s'inquiétèrent des excès de ce "Torquemada de la médiocratie ${ }^{1}$ », des possibles conséquences politiques de tel "phénomène » (le mot courut de débat en éditorial, d'analyse en interview), et s'effrayèrent du brutal Vaffanculo, tel l'Éditorialiste en chef, Eugenio Scalfari, fondateur de La Repubblica: démagogie, populisme, et bientôt fascisme rénové, ou plutôt upgraded, c'est la vérité dévoilée par le juron fait devise, et c'est un dictateur à venir que préparerait sans le savoir la mégalomanie de Grillo, Porte-Parole autoproclamé et acclamé par des masses dont le désir profond (Scalfari dixit) est évidemment d'être « déresponsabilisées ${ }^{2} » \ldots$ Le Comique n'est alors au fond que le pur reflet de son ennemi, l'actuel Président du Conseil, celui que Grillo appelle le «psiconano »-le « nain psychotique ». Bien sûr l'antiparlementarisme, ou plutôt « antipartisme » de Grillo évoque Poujade à des yeux français, tout comme le refrain bien connu du « tous pourris », l'opposition récurrente du « Nous », les petits, et du «Eux» les puissants, et la rhétorique enflammée de Grillo:

L'Italie n'est pas une démocratie. C'est une télécratie, une oligarchie, une mafiocratie. Tout cela à la fois, mais pas une démocratie ${ }^{3}$.

Son Verbe est violence: de la diatribe au réquisitoire, il puise dans tout le répertoire polémique, et ne recule jamais devant l'invective la plus outrancière. Et l'on songe à Scalfari lorsque Grillo tempête contre la « bombe à retardement » que représente l'immigration rom, et l'« insécu-

\footnotetext{
1. C'est le journaliste de La Repubblica Massimo Giannini qui le surnomme ainsi, dans son article « L'ultimo insulto a Napolitano » (« La dernière insulte à Napolitano », NDT: actuel Président de la République italienne), La Repubblica du 26/07/08.

2. Eugenio Scalfari, «L'invasione barbarica del Comico » (« l'Invasion barbare du comique »), $L a$ Repubblica du 12/09/07, en ligne à l'adresse: http://ricerca.repubblica.it/repubblica/archivio/repubblica/2007/09/12/invasione-barbarica-del-comico-grillo.html (consulté le 14 février 2009, comme les citations qui suivent).

3. http://www.beppegrillo.it/2008/11/democrazia_e_no.
} 
rité » corollaire, « milliers de messages » à l'appui. Seulement il ne fait que s'agréger, par telles insanités, au très nauséabond consensus régnant dans l'establishment politique, tous camps inclus, prouvant par là, on y reviendra, que sa position marginale et dissensuelle est toute relative. Tenons alors que Scalfari se fourvoie. Mais sans doute est-il troublé par les gentillesses que Grillo réserve à la presse :

Quand est-ce arrivé? À quel moment les journalistes se sont-ils transformés en tapineurs? Les directeurs de journaux en managers publicitaires? Les rédactions en attachées de presse des grandes entreprises? ça a toujours été comme ça? L'information a toujours été une grande pute ${ }^{1}$ ?

Soit un nouvel épisode de la guerre entre Blogueurs et Éditorialistes: Grillo va jusqu'à publier en version téléchargeable la "Semaine » du blog, dont il signe, bien sûr, l'éditorial. La presse est pour lui l'un de ces parasites (à éliminer, donc) qui entravent l'avènement de la démocratie directe promise par le Réseau. Que le jugement des journalistes soit altéré par ce conflit ou non, il demeure que leur analyse de l'effet-Grillo est souvent lacunaire: Vis comica, parole débridée du Blogueur, rage du Polémiste en sont trois piliers manifestes, mais il en est un quatrième à ne pas sous-estimer. Le crédit de Grillo auprès des foules tient aussi à l'exactitude de ses diagnostics et accusations en plusieurs occasions, à propos des escroqueries tarifaires des compagnies téléphoniques, ou plus encore quant à la banqueroute, par lui seul annoncée, de Parmalat, qui se vérifie peu après, et déclenche un énorme scandale financier. Or Grillo n'a bénéficié de nulle information secrète, ce qu'il précisera aux enquêteurs qui l'interrogeront par la suite: il s'est contenté d'examiner avec attention les documents disponibles, consultables par tout un chacun. Beppe Grillo s'illustre donc aussi comme Contre-Expert, qui se réapproprie les savoirs soi-disant réservés et en fait un usage démocratique. C'est un homme de dossiers (et ancien comptable), qui étudie minutieusement les cas sur lesquels il se penche. Du reste Grillo se nourrit toujours des travaux de Spécialistes, qu'il s'agisse d'écologie ou d'économie, auxquels il se réfère fréquemment, tel Joseph Stiglitz (qui intervient à plusieurs reprises sur le blog). Car les Spécialistes s'intéressent aussi à Grillo, tel le très docte 
et très modéré politologue Giovanni Sartori, spécialiste des institutions démocratiques, qui afficha publiquement son soutien à Grillo ${ }^{1}$.

Si l'on veut vraiment identifier l'idéologie politique grillista, l'hypothèse du pré-fascisme rampant étant écartée, on la dira strictement libérale, (préoccupée en premier lieu des libertés individuelles, en guerre contre tout ce qui s'emploie à les limiter), résolument écologiste, modérément anarchiste (méfiance face à tout corps constitué), et hautement moralisatrice. Reste l'étiquette la plus usuellement assignée à Grillo, l'antipolitique. Là encore, il faut disconvenir. « Antipolitique » se déplie en anti-establishment, anti-partis, anti-gouvernemental. Se découvre ici - chez ceux qui emploient le terme - une conception purement institutionnelle et fort restrictive de la politique. Politique, Grillo l'est, incontestablement, comme les problèmes qu'il soulève, n'en déplaise à ceux qui veulent les esquiver ou qui les créent. Antipolitique, il faudra au contraire lui reprocher de ne pas l'être assez (au sens que lui donnent les détracteurs de Grillo), et mesurer l'écart entre sa férocité verbale et le caractère raisonnable, voire timide, des remèdes préconisés et des mesures souhaitées. Grillo milite pour une moralisation des institutions et de ceux qui les dirigent, pour la transparence de l'État, de ses décisions, de son administration, pour une participation active des citoyens responsables dans la chose publique (il a annoncé la constitution de « listes civiques », indépendantes, aux prochaines élections locales, afin que les citoyens se réapproprient le pouvoir local). Les propositions de loi qu'il défendait en hurlant lors du $V$-day concernent la limitation du nombre de mandats, l'interdiction de siéger au Parlement pour qui a fait l'objet d'une condamnation pénale définitive, la substitution du scrutin uninominal au scrutin de liste. Modifications techniques, exigences morales, revendications calculées. Les tempêtes soulevées par sa voix nasillarde, les foules de grillini galvanisés, l'effroi d' ' intellectuels » alarmés placent Grillo sur le même plan, selon l'analyste, qu'un Le Pen ou qu'un José Bové; ses ambitions législatives le rapprochent pourtant plus d'un Arnaud Montebourg ou d'un François Bayrou.

\footnotetext{
1. Dans son article, « La terra trema sotto la casta » (« la terre tremble sous les pieds de la Caste »; le terme est emprunté au titre d'un livre de deux journalistes du Corriere della sera dénonçant les privilèges d'une « caste » politico-médiatique dominante), Il Corriere della Sera du 19/09/07, en ligne à l'adresse http:/www.corriere.it/Primo_Piano/Editoriali/2007/09_Settembre/19/sartori_grillo_attacca. shtml (consulté le 14/02/09).
} 


\section{Labyrinthe, $n^{\circ} 32$}

Il est convenu aujourd'hui, dans la presse italienne, de décréter le «phénomène Grillo » en phase crépusculaire: rien ne permet une telle certitude, comme rien ne permet encore de dessiner les formes et les chemins qu'empruntera ledit phénomène dans les années à venir. On peut en revanche estimer que la parole de Beppe Grillo n'est sans doute aujourd'hui en Italie ni la moins pertinente, ni la plus inquiétante. 